\title{
Éric Chevillard dans tous ses états, O. Bessard-Banquy et P. Jourde (dir.)
}

\section{Gianmaria Finardi}

\section{(2) OpenEdition}

1 Journals

\section{Edizione digitale}

URL: http://journals.openedition.org/studifrancesi/10068

DOI: 10.4000/studifrancesi. 10068

ISSN: 2421-5856

\section{Editore}

Rosenberg \& Sellier

\section{Edizione cartacea}

Data di pubblicazione: 1 août 2017

Paginazione: 403-404

ISSN: 0039-2944

\section{Notizia bibliografica digitale}

Gianmaria Finardi, «Éric Chevillard dans tous ses états, O. Bessard-Banquy et P. Jourde (dir.)», Studi

Francesi [Online], 182 (LXI | II) | 2017, online dal 01 août 2017, consultato il 11 janvier 2021. URL: http:// journals.openedition.org/studifrancesi/10068; DOI: https://doi.org/10.4000/studifrancesi.10068

Questo documento è stato generato automaticamente il 11 janvier 2021.

\section{(c) (i) (9)}

Studi Francesi è distribuita con Licenza Creative Commons Attribuzione - Non commerciale - Non opere derivate 4.0 Internazionale. 


\title{
Éric Chevillard dans tous ses états, O. Bessard-Banquy et P. Jourde (dir.)
}

\author{
Gianmaria Finardi
}

\section{NOTIZIA}

Éric Chevillard dans tous ses états, sous la direction de Olivier BESSARD-BANQUY et Pierre JOURDE, Paris, Classiques Garnier, 2015, 274 pp.

1 Questa miscellanea, che ha per oggetto l'opera di Éric Chevillard, raccoglie gli atti del convegno internazionale tenutosi a Valence presso la sede distaccata dell'Università di Grenoble 3, nel marzo 2013. Nell'Introduction (pp. 7-14), secondo uno studio diacronico, Olivier BESSARD-BANQUY e Pierre JOURDE passano in rassegna le opere costitutive del nutrito corpus chevillardiano, sottolineando la complessiva poliedricità della poetica di una delle voci preminenti e più spiazzanti dell'attuale panorama letterario.

2 Non a caso il contributo inaugurale, Chevillard, un traitre parmi les traîtres (pp. 17-22), è affidato a CLARO; con molta ironia, lo stravagante scrittore immagina l'autore al centro di improbabili casi di plagiats par anticipation. Procedendo per assurdo, egli arriva a dimostrare l'impossibilità di ridurre a un'etichetta l'opera chevillardiana, da parte di una critica che si avvalesse dei tradizionali strumenti interpretativi.

Successivamente, in La mauvaise foi d'Éric Chevillard (pp. 23-37), Claude cosTE analizza una selezione di quattro romanzi. Si evidenzia la necessità del ricorso alla "malafede", che consente al jeune auteur de Minuit di sottrarsi a un déjà là culturale e a tutte le forme necrotizzate della lingua comune. Assumendo il partito preso dell'ironia, a oltranza, l'autore, che si esprime in modalità squisitamente autofinzionale, può ancora sperare non solo di raccontarsi, ma anche di fare una qualche presa sul reale.

Nel terzo articolo, La littérature sans littérature (pp. 39-50), Alexandre GEFEN propone una lettura metatestuale dei romanzi. Più di ogni altro contemporaneo, l'autore di Préhistoire si fa portavoce di una letteratura non tanto transitiva, quanto riflessiva, quindi votata a una duplice interrogazione. Da una parte, essa sonda la propria 
necessità, fondata nella stessa verve che la anima; d'altra parte, solo accettando di esibire e smentire le proprie assiologie, negando qualsivoglia categoria e visione teleologica, la scrittura può ancora raccontare la realtà, per frammentaria e variabile che sia.

Con Portrait de l'écrivain en animal polymorphe (pp. 39-50), Fabrice THUMEREL punta l'attenzione sul bestiario chevillardiano. Se, in controcorrente rispetto al romanzo realista, l'animale occupa una posizione preminente, ciò si deve alla sua endemica singolarità: grazie a una lingua animale, capace di disarticolare quella comune, l'autore può occupare una posizione altra e altér(is)ante, utile alla causa ontologica. Questa metafora animalière, quindi, rende conto non solo del carattere insolito della poetica, ma anche della sociogénèse dello scrittore.

In Éric Chevillard au Mali (pp. 63-72), studio dedicato a Oreille rouge, Christine JÉRUSALEM sonda il rapporto pastichant dell'autore rispetto ai vari generi letterari. Tale romanzo si dà come vrai faux-récit di un viaggio in Mali, capace di riscrivere ex novo i clichés inerenti all'Africa nera e al racconto di viaggi, non senza ricordare Bruce Chatwin. Questa mimesi formale mostra la velleità del progetto di scrittura del protagonista, alle prese con un'Africa del tutto immaginaria, e permette a Chevillard di interrogarsi sull'autenticità di tanta letteratura che si vorrebbe etnologica.

7 Tocca quindi a Marc DANIEL, in Éric Chevillard et le conte (pp. 73-82), esplorare l'ambigua relazione tra Chevillard e la forma del racconto. Si assiste alla coesistenza di due registri, quali la carnavalisation del genere, operata grazie alla parodia e all'ironia, e la connivenza, condivisa dall'autore con la naturale vocazione del récit che $\mathrm{fu}$ di Maupassant, ovvero il desiderio di situarsi lontano dalla convenzione realista. Ma ecco il vero coup de théâtre del racconto secondo Chevillard: alla ricreazione fantasiosa del mondo si sostituisce un'istantanea loufoque.

8 L'analisi di Laurent DEMANZE, Meurtre en bas de page (pp. 83-92), punta il focus sulla finta recensione letteraria, L'œuvre posthume de Thomas Pilaster, e si prefigge di misurare la portata dell'erudizione. Si tratta di una finzione filologica, il cui afflato narrativo - che presto sfocia in tensione omicida - scaturisce direttamente dalle pratiche incongrue di un savoir faire linguistico, atto a manipolare e sopprimere la positività del sapere come comunemente è inteso. In ciò consiste una delle principali trappole del romanzo, teso a gettare l'ombra del sospetto sull'atto stesso della lettura.

9 In Espaces du renversement et de l'inachèvement (pp. 93-106), Aurélie ADLER mette in luce il carattere atemporale dei testi. Partendo da una cernita di tre romanzi, la studiosa isola altrettanti territori critici dove l'autore rovescia le utopie progressiste, declinate nello spazio antistorico della grotta di Préhistoire, in quello ucronico dell'estinzione animale di Sans l'orang-outan o nell'isola distopica di Choir. Mentre fa implodere l'ideale umanista, reinventando spazi per l'alterità, Chevillard spinge sull'acceleratore di un immaginario apocalittico ma eternamente rinnovato, senza mai portarlo davvero a fine corsa.

10 L'articolo di Marie-Odile ANDRÉ, Palafox au pays des moralistes (pp. 107-120), mostra come Chevillard adotti, tanto nelle opere quanto nel suo fortunato blog L'autofictif, una postura da moralista sui generis che poggia su tre assi principali: un'autorità enunciativa che si ricompone nel movimento stesso del proprio disfacimento, un racconto che si sviluppa nel tentativo di farsi frammentario, delle scelte etiche che si fondano sulla parte più umorale del giudizio. Non a caso, anche quanto a questa complessiva 
instabilità posturale, la poetica può essere incarnata da Palafox, lo sfuggente animale protagonista dell'eponimo romanzo.

11 Il decimo intervento, La disposition du grognon (pp. 121-136), è interamente dedicato a L'auteur et moi, testo in cui l'autore non esita a interrompere il suo personaggio per raccontare la propria storia. Béatrice BLOCH isola un intero campionario di procedimenti, come il renversement parodico dei generi codificati, la presentazione di teorie infantili, il ricorso al delirio logico e l'assunzione di un tono pince-sans-rire. Le materie costitutive di questo patchwork testimoniano tutte della volontà di giocare con il linguaggio e restituiscono al lettore il piacere della gratuità, l'esperienza della libertà. In Virulence de Chevillard (pp. 137-150), Jean-Bernard VRAY individua la figura di un narratore «jubilatoirement violent» sempre al centro dei testi, ricorrendo alla categoria estetica ed enunciativa della virulence. Lo studioso osserva quindi tutta la varietà di strumenti utilizzati da Chevillard per guastare il cosiddetto meccano del racconto. Infine dimostra non solo il carattere autografico - ben prima che autobiografico - delle opere in questione, ma soprattutto la legittima sovrapposizione dell'ethos del narratore a quello dell'autore, perennemente finzionalizzato e travolto nella pulsione testofagica della scrittura.

13 Con Énumérations dilatoires (pp. 151-168), Gaspard TURIN ribadisce il carattere digressivo dei testi, che tendono ora a inceppare il progredire del récit, ora verso un'espansione dello stesso; resta da comprendere se nel romanzo chevillardiano esista un fondo su cui poggia tale scrittura dilatoria o se invece si debba parlare di doppio, di triplo fondo. $\mathrm{Si}$ individuano quindi nei procedimenti della lista e del cliché la sintesi perfetta delle opposte forze che governano i testi: da un lato, in negativo, il rifiuto del bon vieux roman, dall'altro - grazie al coinvolgimento propositivo, quando non metalettico, del lettore - il reinvestimento degli stessi valori romanesques. Questo duplice diversivo costituisce una possibile base dell'estetica chevillardiana.

14 L'articolo di Lia KURTS-KOSTE, La dynamique énonciative dans les récits d'Éric Chevillard (pp. 169-192), analizza il funzionamento delle principali configurazioni enunciative dei romanzi - dai commenti metadiscorsivi alle rotture di tono e delle catene referenziali, passando per i pastiches, per arrivare all'eterofonia -, concentrandosi sul caso di Palafox. $\mathrm{Ne}$ emergono l'apologia e l'illustrazione aporetiche di una letteratura, che, perennemente divisa tra un potere etico e uno estetico, persegue l'obiettivo di liberare dalla fissità le forme della lingua e quelle dell'immaginario.

In L'autofictif observe le monde (pp. 193-214), Pascal RIENDEAU osserva innanzitutto come l'autore, nei volumi de L'autofictif, cancelli la tradizionale linea di demarcazione tra frammento letterario e aforisma, puntando sulla creazione di una forma breve tanto ibrida quanto inedita. In seguito, lo studioso esplora la sfaccettata visione del mondo restituitaci da Chevillard a suon di commenti sagaci e ironici, la stessa visione che fa del partito preso della discontinuità non solo un modo efficace per «piéger la bonne conscience» e le posizioni preconcette, ma addirittura una questione etica.

In Chevillard lecteur (pp. 215-226), Anne RocHE inizia con l'osservare il ruolo centrale del lettore all'interno dei romanzi, convocato sotto forma di personaggi, situazioni e strutture. Del resto, anche quando si trova dall'altra parte della barricata, nei panni del cronista - nelle declinazioni del critico-feuilletoniste per «Le Monde» e del blogger -, egli non esita a sparare a vista sugli autori che non apprezza, ritenuti i rappresentanti di 
una letteratura asservita all'ordine costituito, né a correre in soccorso di quelli rimasti incompresi, a cui la critica non ha ancora riservato il giusto riconoscimento.

Il contributo di Blanche CERQUIGLINI, L'auteur en entretien (pp. 227-241), ha per oggetto il corpus delle interviste concesse dallo scrittore negli ultimi venticinque anni. Lungi dal risultare un mero complemento, questi testi fanno pienamente parte dell'opera romanesque. Da un lato, infatti, si danno come spazi liberi, che consentono a Chevillard di sfoderare tutte le proprie armi stilistiche; dall'altro, concorrono a tracciare il ritratto di un autore in collera, che agogna una radicale riforma della lingua e una profonda trasformazione del mondo, sabotando, dall'interno, la Letteratura.

Nell'ultimo intervento, Éric Chevillard par la presse (pp. 242-257), Ekaterina KOULECHOVA si prefigge di indagare il contesto editoriale immediato dell'opera di Chevillard. Da una scelta di critiche letterarie e di interviste inedite di giornalisti emerge un quadro paradossale: si tratterebbe sì di una voce incontournable, ma anche di una voce a parte, considerata pertanto da molti cerebrale, ermetica, "difficile" insomma. Anche così, si delinea il profilo atipico dello scrittore inclassificabile, la cui opera relativamente giovane non ha forse ancora raggiunto il grande pubblico, ma è già entrata a far parte dei programmi scolastici.

Una menzione particolare merita il testo inedito, Le colloque pour tous (pp. 15-16), appositamente scritto da Éric CHEVILLARD per celebrare da par suo il convegno stesso, al quale aveva per altro assistito di persona. Qui, con una gran dose di (auto)ironia, una voce narrante molto vicina a quella dell'autore auspica che un giorno tutti gli abitanti del pianeta possano diventare l'oggetto di un convegno internazionale: solo allora «les ténèbres se dissiperont, l'oblique rayon du soleil traversera le cristal de nos crânes pour caresser le galbe de ce globe comme au premier jour». 